\title{
Bioavailability of Acetate from Two Vinegar Supplements: Capsule and Drink
}

\author{
Shino SugiYama ${ }^{1}$, Takashi Fushimi ${ }^{1, *}$, Mikiya KISHI $^{1}$, Shin IrIE ${ }^{2}$, Shigeki TsujI ${ }^{2}$, \\ Natsuko HoSOKAWA ${ }^{3}$ and Takayuki KAGA ${ }^{1}$ \\ ${ }^{1}$ Central Research Institute, Mizkan Group Corporation, Handa 475-8585, Japan \\ ${ }^{2}$ Nishikumamoto Hospital, Medical Co. LTA, Kumamoto 861-4157, Japan \\ ${ }^{3}$ Clinical Research Department, Mediscience Planning Inc., Tokyo 103-0004, Japan
}

(Received January 20, 2010)

\begin{abstract}
Summary The bioavailability of acetate in various vinegar supplements, e.g. as capsules and drinks, remains unclear. Thus, we conducted a cross-over clinical study in 30 healthy subjects. After an overnight fast, subjects received each test sample in a randomised sequence: 9 vinegar capsules (containing $750 \mathrm{mg}$ acetic acid in total) with $150 \mathrm{~mL}$ of water, $100 \mathrm{~mL}$ of vinegar drink (containing $750 \mathrm{mg}$ acetic acid), and $150 \mathrm{~mL}$ of water as reference. Blood samples were collected before (defined as $0 \mathrm{~min}$ ), at 15, 30, 45, 60, 90, 120 and 180 min after each test sample intake. In the vinegar drink group, serum acetate concentration increased immediately after intake, peaked at $15 \mathrm{~min}$ and returned to baseline at $90 \mathrm{~min}$. That in the vinegar capsule group rose slowly, peaked at $30 \mathrm{~min}$ and returned to baseline at $120 \mathrm{~min}$. The peak values in both groups exceeded $200 \mu \mathrm{mol} / \mathrm{L}$, the physiologically active concentration confirmed by in vitro experiment. In the reference group, levels remained constant throughout the 180-min period. The amount of absorbed acetate from the vinegar capsule group and the drink group was evaluated by the difference value of the area under the serum acetate concentration-time curve (AUC) between in each vinegar group and in the reference group (expressed as $\mathrm{AUC}_{\text {capsule-ref }}$ and $\mathrm{AUC}_{\text {drink-ref, }}$ respectively). AUC capsule-ref $_{\text {was }}$ about $80 \%$ of $A U C_{\text {drink-ref, }}$ but there was no significant difference between them.
\end{abstract}

Key Words vinegar, acetate, supplements, absorption, bioavailability

Vinegar, whose main component is acetic acid, is an important seasoning in use all over the world. In the past decade, continuous intake (on a daily basis) of a drink containing $15 \mathrm{~mL}$ vinegar $(750 \mathrm{mg}$ acetic acid) was reported to improve lifestyle-related diseases such as hypertension (1), hyperlipidaemia (2) and obesity (3). Furthermore, animal studies demonstrated that acetic acid was the active ingredient responsible for these effects (4-9).

Acetic acid is absorbed after the consumption of a meal containing vinegar (10), and then circulates in the acetate form to be metabolized by the whole body (11). The effects of vinegar intake on the various lifestyle-related diseases are due to this acetate form, and the proposed mechanisms are as follows: anti-hypertension, enhancement of vasodilatation through adenosine receptor by acetate in blood vessels (12); anti-hyperlipidaemia and anti-obesity, inhibition of lipogenesis and enhancement of fatty acid oxidation via activation of AMP-activated protein kinase (AMPK) by acetate in the liver (5, 7-9). Therefore, to enjoy the benefits of vinegar, it is important that acetate is adequately absorbed by the body and that serum acetate levels rise above

*To whom correspondence should be addressed.

E-mail: tfushimi@mizkan.co.jp background levels.

There are various forms of vinegar supplements on the market, e.g. capsules and drinks. When it come to capsules, though, not all vinegar capsules contain an adequate amount of acetate. Moreover, there have been case reports of non-absorption of the active ingredient from such capsules $(13,14)$. Although, as discussed above, absorbed acetate plays an important role in the physiological functioning of vinegar, nevertheless, there have been no reports to date documenting the bioavailability of acetate after the intake of commercial vinegar supplements. Therefore, we considered it valuable to assess the change in serum acetate concentration over time after the intake of our products. Therefore, we report on our investigation into acetate bioavailability from both the vinegar capsule and the vinegar drink, using a cross-over, clinical study in healthy Japanese subjects.

\section{Materials and Methods}

Subjects. Thirty healthy Japanese subjects (17 men and 13 women) were enrolled in this study. Their ages ranged from 23 to $59 \mathrm{y}(40.4 \pm 2.2)$. None had a history of alimentary allergy or serious disease, and none were currently taking any medication. Written informed consent for participation was obtained from all subjects. 
The Ethics Committees of the Mizkan Group Corporation and Nishikumamoto Hospital approved the study protocol and found it to be in accordance with the Declaration of Helsinki.

Test samples. There were three types of test samples: $150 \mathrm{~mL}$ of non-carbonated mineral water (Suntory Natural Water, Suntory Foods Co., Ltd., Tokyo, Japan), which acted as the reference group [Sample A]; a bottle $(100 \mathrm{~mL})$ of the vinegar drink, approved as Food for Specified Health Uses (MAINZ, Mizkan Co., Ltd., Aichi, Japan) [Sample B]; or 9 vinegar capsules (MICARA, Mizkan Group Corporation, Aichi, Japan) with $150 \mathrm{~mL}$ of the reference non-carbonated mineral water [Sample C]. One hundred milliliters of the vinegar drink and 9 vinegar capsules contained $750 \mathrm{mg}$ acetate [the other nutritional composition is as follows: energy (kJ) 66.9, 125.5 , carbohydrates $(\mathrm{g}) 3.9,1.02$, fat $(\mathrm{g}) 0,2.28$, protein $(\mathrm{g})$ 0, 1.56 and calcium (mg) 65, 300 in the drink or the 9 capsules, respectively].

Study design. This was a single-blind, randomized cross-over study, performed between July and September 2007. The investigators were blinded to the treatments. To examine acetate bioavailability twice (the first and the second set) per test sample, six test periods were included in this study. Therefore, the 30 subjects were randomly assigned to one of six sequences: 1) A-B$\mathrm{C} \times 2$, 2) $\mathrm{B}-\mathrm{C}-\mathrm{A} \times 2,3) \mathrm{C}-\mathrm{A}-\mathrm{B} \times 2$, 4) $\mathrm{A}-\mathrm{C}-\mathrm{B} \times 2$, 5) $\mathrm{C}-\mathrm{B}-$ $\mathrm{A} \times 2$, and 6) $\mathrm{B}-\mathrm{A}-\mathrm{C} \times 2$. The washout interval between the first and the second test periods was 6-13 d. On the night preceding the test period, the subjects ate a "study supper," which they had to consume before 21:00 h. Following their supper, the subjects fasted until after the 180-min blood sample had been taken the following day. In addition, they were instructed not to consume any alcohol on the day preceding the test period. The "study supper" consisted of $130 \mathrm{~g}$ of rice (Sato-nogohann Karukuichizen, Sato Foods Co., Ltd., Niigata, Japan) and $270 \mathrm{~g}$ of a ready-prepared meal (Kikubarigozen Gomokudofu-no-ankake set, Nichirei Foods Direct, Inc., Tokyo, Japan). The total nutritional content of the supper was as follows: energy $(\mathrm{kJ}) 2,160$, carbohydrates (g) 76.5, fat (g) 15.0 and protein $(\mathrm{g})$ 18.7. On the morning of the test period, subjects were to arrive at Nishikumamoto Hospital by 08:00 h. After the subject had rested for $30 \mathrm{~min}$ on a bed, a catheter was inserted in one arm, and the relevant test sample was taken within $3 \mathrm{~min}$. Blood samples $(4 \mathrm{~mL})$ were immediately drawn from the antecubital vein into a glass vacuum tube before (defined as $0 \mathrm{~min}$ ), at 15, 30, 45, 60, 90, 120 and $180 \mathrm{~min}$ after the test sample intake. Blood samples were stored at room temperature for $30 \mathrm{~min}$, and then the tubes were centrifuged at 3,000 rpm for $10 \mathrm{~min}$. The serum was immediately transferred to a screw-capped tube, and stored at $-20^{\circ} \mathrm{C}$ until analysis. Subjects remained at rest on their bed in a quiet and airconditioned room (a constant temperature of $26-27^{\circ} \mathrm{C}$ ) until after the 180-min blood sample had been collected. Water was freely available except $30 \mathrm{~min}$ before and after the test sample intake.

Measurement of serum acetate levels. Serum acetate levels $(\mu \mathrm{mol} / \mathrm{L})$ were measured by gas chromatography with isovaleric acid as an internal standard (15). The chromatograph was a GC-2010 (Simadzu, Kyoto, Japan). All reagents for the measurement were purchased from Wako Pure Chemical Industries, Ltd. (Osaka, Japan).

Statistical analysis. All data except nutrient composition are shown as mean \pm SE. The transitions of serum acetate concentrations in the first set of each treatment were the same as those in the second set. Therefore, the averaged value of the two sets was analyzed. The area under the serum acetate concentration-time curve [AUC $(\mu \mathrm{mol} \times \min / \mathrm{L})]$ was calculated by the linear trapezoidal rule. The amount of absorbed acetate from the vinegar capsule group and the drink group was evaluated by the difference value of AUC between in each vinegar group and in the reference group (expressed as $\mathrm{AUC}_{\text {capsule-ref }}$ and $\mathrm{AUC}_{\text {drink-ref, }}$ respectively).

Serum acetate levels in the reference group remained constant throughout the 180-min period. Thus, repeated measures multivariate ANOVA was used to examine the effect of treatment over time between the vinegar capsule and the vinegar drink group (SPSS for Windows version 11.5J; SPSS, Inc., Chicago, IL). For inter-group comparisons at each time point, intragroup comparisons in each treatment group and comparisons in AUC among the three groups, one-way repeated measures ANOVA, followed by the Bonferroni test, was used. The difference in the peak value and the amount of absorbed acetate between the vinegar capsule and the vinegar drink groups was analyzed by a paired $t$-test. Differences were considered significant when $p<0.05$.

\section{Results}

Time course of serum acetate concentrations

Serum acetate concentration in the vinegar drink group increased immediately after intake (Fig. 1), while that in the vinegar capsule group rose gradually after intake. The peaks were found at 15 and $30 \mathrm{~min}$, respectively. The peak in the vinegar drink group was significantly higher than in the vinegar capsule group $(349 \pm 21$ vs. $216 \pm 14, p<0.001)$. There was a significant interaction (time $\times$ treatment) between the vinegar capsule and the vinegar drink group $(p<0.001)$. The serum acetate levels in the reference group remained stable throughout the 180-min measurement period. Both the vinegar drink group and the vinegar capsule group had higher serum acetate levels than the reference group at 15-60 min, and at 30-90 and $180 \mathrm{~min}$, respectively. The levels in the vinegar drink group were significantly higher than those in the vinegar capsule group at $15 \mathrm{~min}(349 \pm 21$ vs. $142 \pm 7, p<0.001)$ and $30 \min (265 \pm 12$ vs. $216 \pm 14, p=0.013)$, but were significantly lower at $90 \mathrm{~min}(131 \pm 5$ vs. $157 \pm 6, p=$ 0.008). Both the vinegar drink group and the vinegar capsule group had higher serum acetate levels than time 0 , at $15-45 \mathrm{~min}$, and at 30-90 min, respectively. Serum acetate concentrations in the vinegar drink group and in the vinegar capsule group returned to 


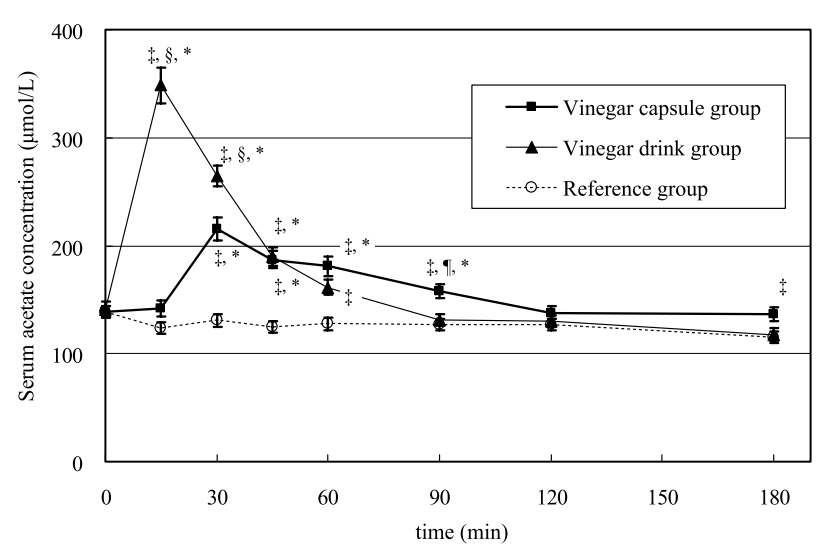

Fig. 1. Time-course changes of serum acetate concentration in the vinegar drink group, the vinegar capsule group and the reference group. Individual values are the means of values for each test sample repeated twice. Data points are means with their standard errors shown by vertical bars. There was a significant interaction (time $\times$ treatment) between the vinegar capsule and the vinegar drink group $(p<0.001) . \stackrel{+}{\lessgtr}$ Mean value in the vinegar capsule or the vinegar drink group was significantly higher than that in the reference group $(p<0.05)$. "Mean value in the vinegar capsule group was significantly higher than that in the vinegar drink group $(p<0.05)$. ${ }^{\S}$ Mean value in the vinegar drink group was significantly higher than that in the vinegar capsule group $(p<0.05)$. ${ }^{*}$ Mean value at each time was significantly higher than that at time $0(p<0.05)$. There was no significant difference in the reference group.

their initial value at 90 and $120 \mathrm{~min}$, respectively. Amount of absorbed acetate

$\mathrm{AUC}_{\text {capsule-ref }}$ and $\mathrm{AUC}_{\text {drink-ref, }}$ as indexes of the amount

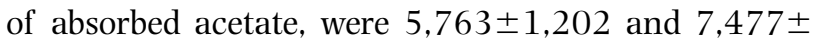
1,067 , respectively. There was no significant difference between them.

\section{Discussion}

The present study is the first to demonstrate that acetate is absorbed after the intake of a vinegar supplement. The significant interaction indicated that the time course of the change of serum acetate levels differed between the capsules and the drink. The acetate absorption began immediately or gradually, after intake of the vinegar drink or capsule, respectively. Although the acetate absorption from the capsules was slower than seen with the vinegar drink, it continued for longer, and consequently $\mathrm{AUC}_{\text {capsule-ref }}$ was about $80 \%$ of AUC drink-ref.

Acetate is considered to be absorbed in the upper digestive tract, especially the stomach and jejunum (16-18), and to be metabolised by the whole body (11). Thus, it was not unexpected that the serum acetate concentration would increase immediately after intake of the vinegar drink. The vinegar capsule used in the present study was covered with soft gelatin. In preliminary examinations, these capsules were found to collapse within $20 \mathrm{~min}$ in the first fluid of the Japanese Pharmacopoeia XIV (artificial gastric juice) using the disintegration test. It has been reported that no difference was observed between the gastric emptying time after intake of acetic acid and acetate. Thus, the start of acetate absorption from the vinegar capsule was delayed relative to the drink, not because of gastric emptying, but because of the protective gelatin coating.

Adenosine and AMP, which are produced when absorbed acetate is metabolised, induce vasodilatation (12) and activation of AMPK (8), respectively. The present study showed that acetate was absorbed by intake of the vinegar supplement and that the amount of absorbed acetate from the vinegar capsule was about $80 \%$ of that from the vinegar drink. Thus, the vinegar supplements might be expected to be beneficial for control of blood pressure and lipid metabolism via production of these two metabolites. Also it was found that serum acetate concentration rose above baseline after intake of both the vinegar drink and the capsules, and that the peak values in both groups were $>200 \mu \mathrm{mol} /$ L. Previous studies $(5,9)$ have reported that $200 \mu \mathrm{mol} /$ $\mathrm{L}$ of acetate reduced mRNA expression of sterol-regulatory element binding protein-1, a fatty acid synthesis enhancer, and increased mRNA expression of peroxisome proliferator-activated receptor $\alpha$ and uncoupling protein 2 , mediators of fatty acid oxidation and thermogenesis, respectively, through activation of AMPK in hepatocytes. These actions result in a reduction of high serum lipid levels and body fat mass. Taken together, these results indicate that the vinegar capsule as well as the vinegar drink might be beneficial for lifestyle-related diseases such as hypertension, hyperlipidaemia, and obesity via the same mechanism.

In conclusion, the vinegar drink and the capsules containing $750 \mathrm{mg}$ acetic acid led to an acute and moderate rise of serum acetate concentration, respectively. The peak of serum acetate levels in both vinegar supplement groups exceeded the physiologically active concentration. The amount of absorbed acetate was not so different between these groups. Therefore, continuous intake of the vinegar capsule is thought to be as useful as the vinegar drink for achieving the health benefits of vinegar for lifestyle-related diseases. It is important to confirm the effect of the vinegar capsules by further clinical studies.

\section{REFERENCES}

1) Kajimoto O, Tayama K, Hirata H, Takahashi T, Tsukamoto Y. 2001. Effect of a drink containing vinegar on blood pressure in mildly and moderately hypertensive subjects. Kenko Eiyo Shokuhin Kenkyu (J Nutr Food) 4(4): 47-60 (in Japanese with English summary).

2) Fushimi T, Ohshima Y, Kishi M, Nishimura A, Kajimoto O, Tsukamoto Y. 2005. Effects of a drink containing vinegar on serum total cholesterol and assessment of its safety. Kenko Eiyo Shokuhin Kenkyu (J Nutr Food) 8(1): 13-26 (in Japanese with English summary).

3) Kondo T, Kishi M, Fushimi T, Ugajin S, Kaga T. 2009. Vinegar intake reduces body weight, body fat mass, and serum triglyceride levels in obese Japanese subjects. Biosci Biotechnol Biochem 73: 1837-1843.

4) Kondo S, Tayama K, Tsukamoto Y, Ikeda K, Yamori Y. 
2001. Antihypertensive effects of acetic acid and vinegar on spontaneously hypertensive rats. Biosci Biotechnol Biochem 65: 2690-2694.

5) Sakakibara S, Yamauchi T, Oshima Y, Tsukamoto Y, Kadowaki T. 2006. Acetic acid activates hepatic AMPK and reduces hyperglycemia in diabetic KK-A(y) mice. Biochem Biophys Res Commun 344: 597-604.

6) Tanizawa H, Sazuka Y, Komatsu (Serita) A, Takino Y. 1983. Acute toxicity of Komezu and its effects on lipid metabolism in male mice. Nippon Eiyo Shokuryo Gakkaishi (J Jpn Soc Nutr Food Sci) 36: 283-289 (in Japanese with English summary).

7) Fushimi T, Suruga K, Oshima Y, Fukiharu M, Tsukamoto Y, Goda T. 2006. Dietary acetic acid reduces serum cholesterol and triacylglycerols in rats fed a cholesterol-rich diet. Br J Nutr 95: 916-924.

8) Yamashita H, Fujisawa K, Ito E, Idei S, Kawaguchi N, Kimoto M, Hiemori M, Tsuji H. 2007. Improvement of obesity and glucose tolerance by acetate in Type 2 diabetic Otsuka Long-Evans Tokushima Fatty (OLETF) rats. Biosci Biotechnol Biochem 71: 1236-1243.

9) Kondo T, Kishi M, Fushimi T, Kaga T. 2009. Acetic acid increases gene expression of fatty acid oxidation enzymes in liver and suppresses body fat accumulation. J Agric Food Chem 57: 5982-5986.

10) Brighenti F, Castellani G, Benini L, Casiraghi MC, Leopardi E, Crovetti R, Testolin G. 1995. Effect of neutralized and native vinegar on blood glucose and acetate responses to a mixed meal in healthy subjects. Eur J Clin Nutr 49: 242-247.
11) Cummings JH, Pomare EW, Branch WJ, Naylor CP, Macfarlane GT. 1987. Short chain fatty acids in human large intestine, portal, hepatic and venous blood. Gut 28: 1221-1227.

12) Carmichael FJ, Saldivia V, Varghese GA, Israel Y, Orrego H. 1988. Ethanol-induced increase in portal blood flow: role of acetate and A1- and A2-adenosine receptors. Am J Physiol 255: G417-G423.

13) Miles MV, Horn P, Miles L, Tanga P, Steelec P, DeGrauwa T. 2002. Bioequivalence of coenzyme Q10 from overthe-counter supplements. Nutr Res 22: 919-929.

14) Leonard SW, Good CK, Gugger ET, Traber MG. 2004. Vitamin E bioavailability from fortified breakfast cereal is greater than that from encapsulated supplements. $\mathrm{Am}$ J Clin Nutr 79: 86-92.

15) Brighenti F. 1997. Simple method for quantitative analysis of short chain fatty acids in serum by gas-liquid chrmotography. In: Plant Polysaccharides in Human Nutrition: Structure, Function, Digestive Fate and Metabolic Affects (Guillon F, Abraham G, Amado R, eds), p 114-119. INRA, Nantes.

16) Schmitt MG Jr, Soergel KH, Wood CM. 1976. Absorption of short chain fatty acids from the human jejunum. Gastroenterology 70: 211-215.

17) Saunders D. 1991. Absorption of short chain fatty acids in human stomach and rectum. Nutr Res 11: 841-847.

18) Argenzio RA, Southworth M. 1975. Sites of organic acid production and absorption in gastrointestinal tract of the pig. Am J Physiol 228: 454-460. 